\title{
Internationale Studienergebnisse
}

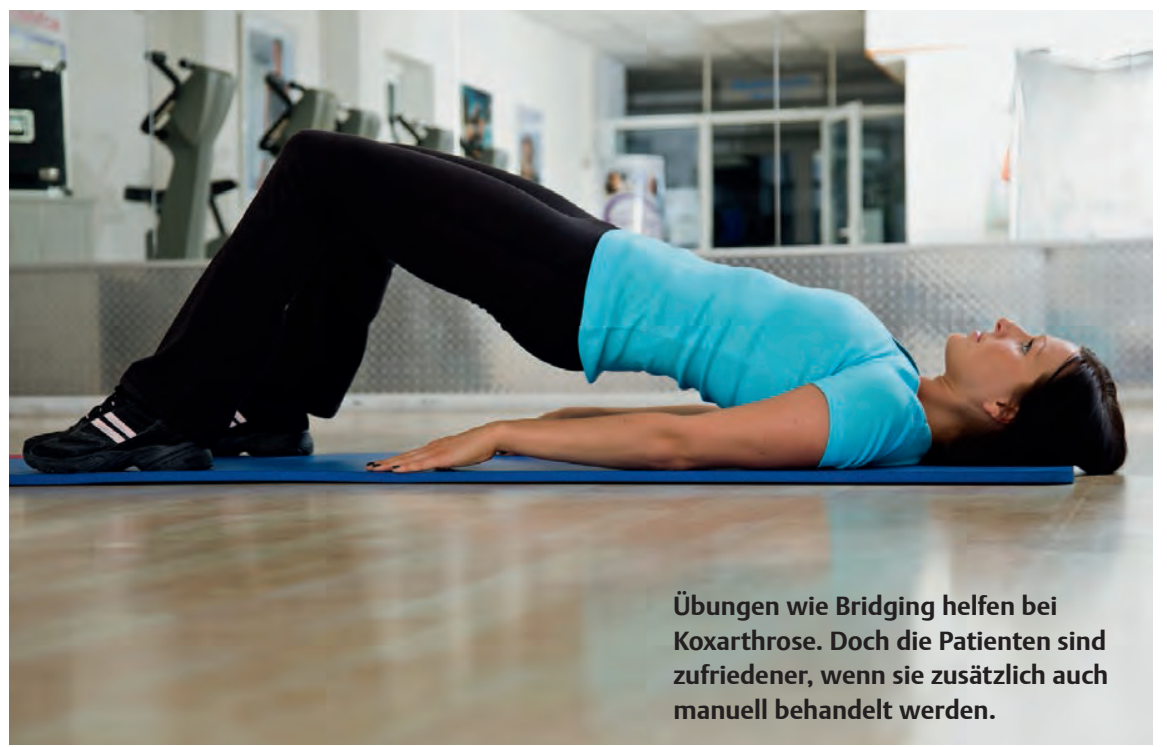

KOXARTHROSE

Prävalenz

Anteil aller Menschen, die im Laufe ihres Lebens eine Koxarthrose entwickeln

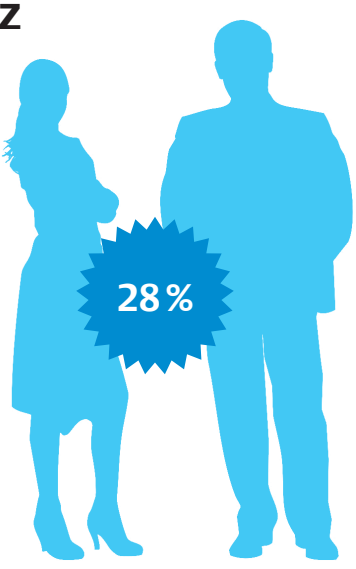

Quelle: Arch Phys Med Rehabil 2013; 94: 302-314

\section{KOXARTHROSE}

\section{Manuell therapierte Patienten sind zufriedener}

Patienten mit Koxarthrose, die zusätzlich zu einem Übungsprogramm Manuelle Therapie erhalten, geht es nicht besser als Patienten, die ausschließlich selbst üben. Aber: Manuell behandelte Patienten sind zufriedener. Zu diesem Ergebnis kam ein Team aus Irland.

Die Forscher schlossen 131 Patienten in ihre Studie ein, die zwischen 40 und 80 Jahre alt waren und klinische und radiologische Zeichen einer Koxarthrose hatten. Die Probanden wurden per Zufall auf drei Gruppen verteilt: Übungtherapie (ÜT), Übungstherapie und Manuelle Therapie (MT), keine Therapie (KT). Die
ÜT- und die MT-Gruppe absolvierten über acht Wochen insgesamt sechs bis acht Therapieeinheiten. Die ÜT bestand aus einem standardisierten, 30-minütigen Programm mit Kraftund Beweglichkeitstraining. Dazu erhielten die Probanden ein Heimprogramm und den Ratschlag, fünfmal pro Woche für je mindestens 30 Minuten ein Ausdauertraining zu absolvieren - zum Beispiel Schwimmen oder Radfahren. Dazu bekamen sie Informationen über Hüftgelenkarthrose. Die MT-Gruppe erhielt zusätzlich 15 Minuten lang manuelle Mobilisationen, wobei maximal fünf Techniken ange- wandt werden durften. Die Kontrollgruppe bekam lediglich die Infos über Koxarthrose.

Nach neun und 18 Wochen konnten die Wissenschaftler zwischen der ÜT- und MTGruppe keinen Unterschied feststellen - weder im primären Outcome-Parameter, dem WOMAC (Western Ontario and McMaster Universities Osteoarthritis Index), noch bei den sekundären, beispielsweise der Hüftgelenkbeweglichkeit. Lediglich die Zufriedenheit mit der Behandlung war in der MT-Gruppe höher als bei den Patienten der ÜT-Gruppe. josc Arch Phys Med Rehabil 2013; 94: 302-314

\section{KRYOTHERAPIE}

\section{Langzeitkühlung verändert Muskeleigenschaften}

- Kühlt man den Quadrizeps 20 Minuten lang, verändern sich seine Eigenschaften. Das fanden Forscher aus Finnland heraus.

Sie applizierten auf dem Oberschenkel von 39 Gesunden einen Kühlpack von 35x29 cm Größe, der im Schnitt knapp über $0^{\circ} \mathrm{C}$ kalt war. Nach 20 Minuten hatten sich die Muskeleigenschaften signifikant verändert: Der Muskel war steifer, angespannter und weniger elastisch als zuvor. Nach 15 Minuten hatten sich die meisten Muskeleigenschaften wieder normalisiert. Dennoch weisen die Wissenschaftler darauf hin, dass es notwendig ist, die Muskulatur nach einer Eisanwendung erst wieder sorgfältig aufzuwärmen.

josc

Phys Ther Sport 2012;

13: $265-269$ 


\section{Unilaterale OP - bilateraler Benefit}

Achillodynien treten häufig beidseits auf. Inzwischen gibt es Vermutungen, dass eine Beteiligung des Zentralnervensystems an den Beschwerden ein Grund dafür sein könnte. Ein Team von Wissenschaftlern um den Achillessehnen-Experten Håkan Alfredson untersuchten daher nun, inwieweit eine unilaterale OP auch die Beschwerden auf der nicht operierten Seite beeinflusst.

An der Studie nahmen 13 Patienten mit chronischer, bilateraler Tendinopathie teil, bei denen eine konservative Therapie, beispielsweise mit Schonung der betroffenen Seite oder exzentrischem Training, erfolglos gewesen war. Für die Operation wählten die Wissenschaftler die Seite aus, die zum Zeitpunkt der Untersuchung am schmerzhaftesten war. Bei dem Eingriff wendeten sie die sogenannte Scraping-Methode an.

Nach einem halben Jahr hatten sich bei elf der 13 Studienteilnehmer die Beschwerden auf beiden Seiten gebessert. Nach 37 Monaten war die Schmerzreduktion bei den elf Patienten signifikant, zudem waren die Teilnehmer mit dem Ergebnis sehr zufrieden. Zehn

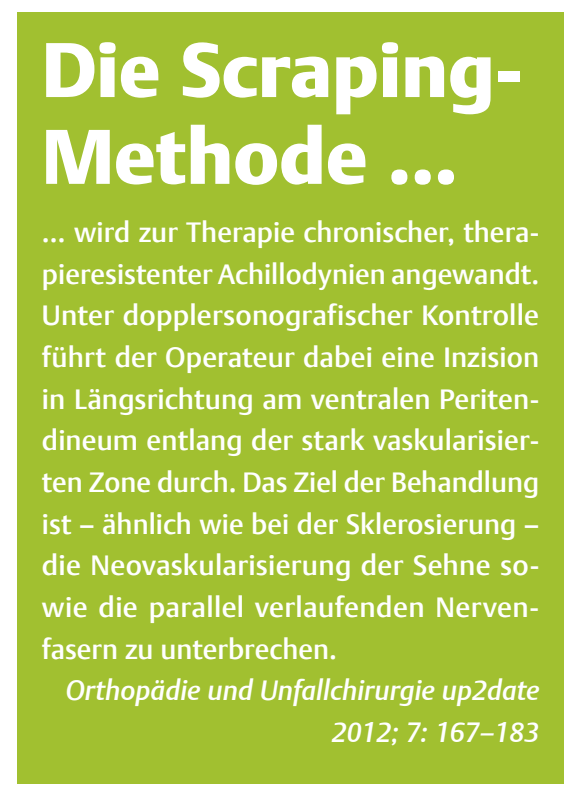

der Patienten waren bei Aktivitäten mit normaler Belastungsintensität sogar vollständig schmerzfrei.

Br J Sports Med 2012; doi: 10.1136/bjsports-2012-091399

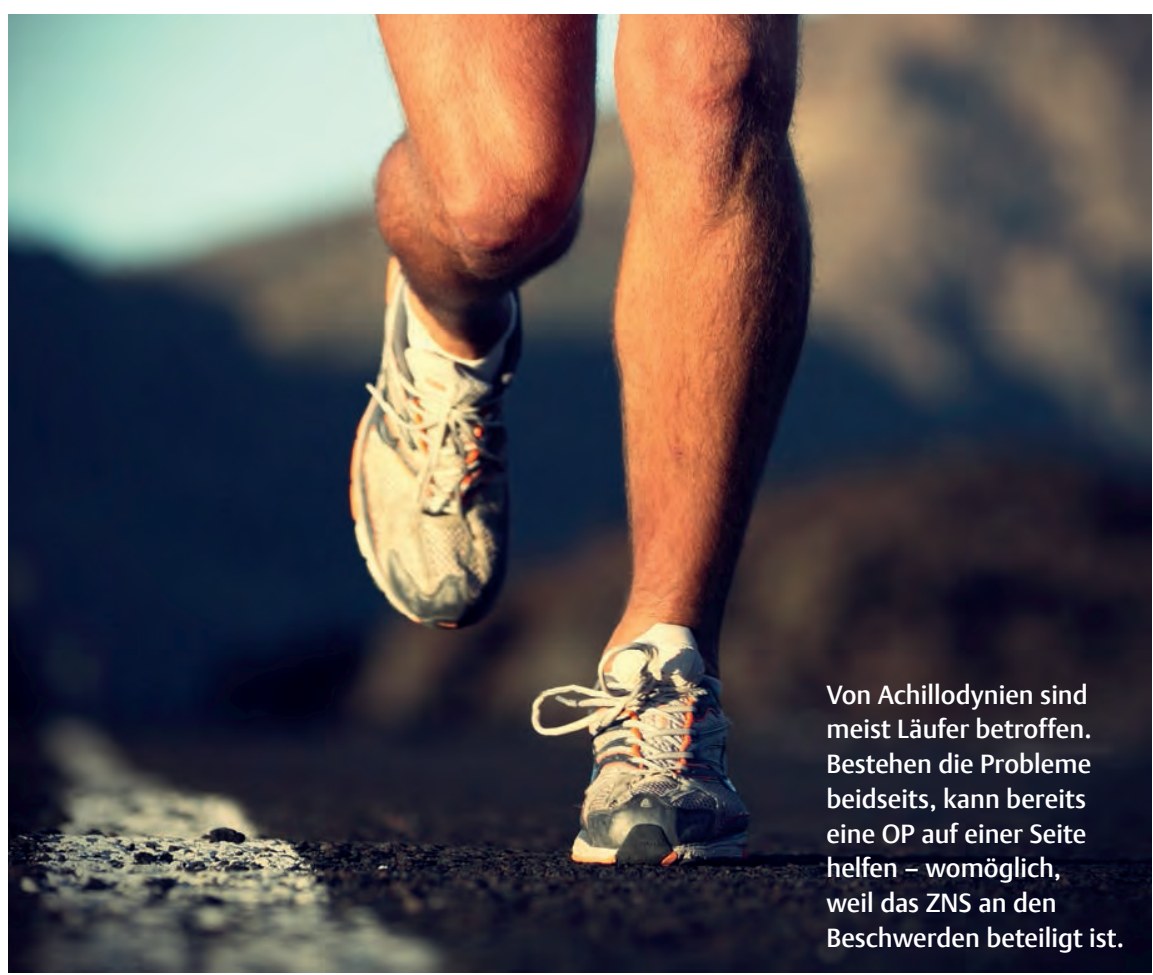




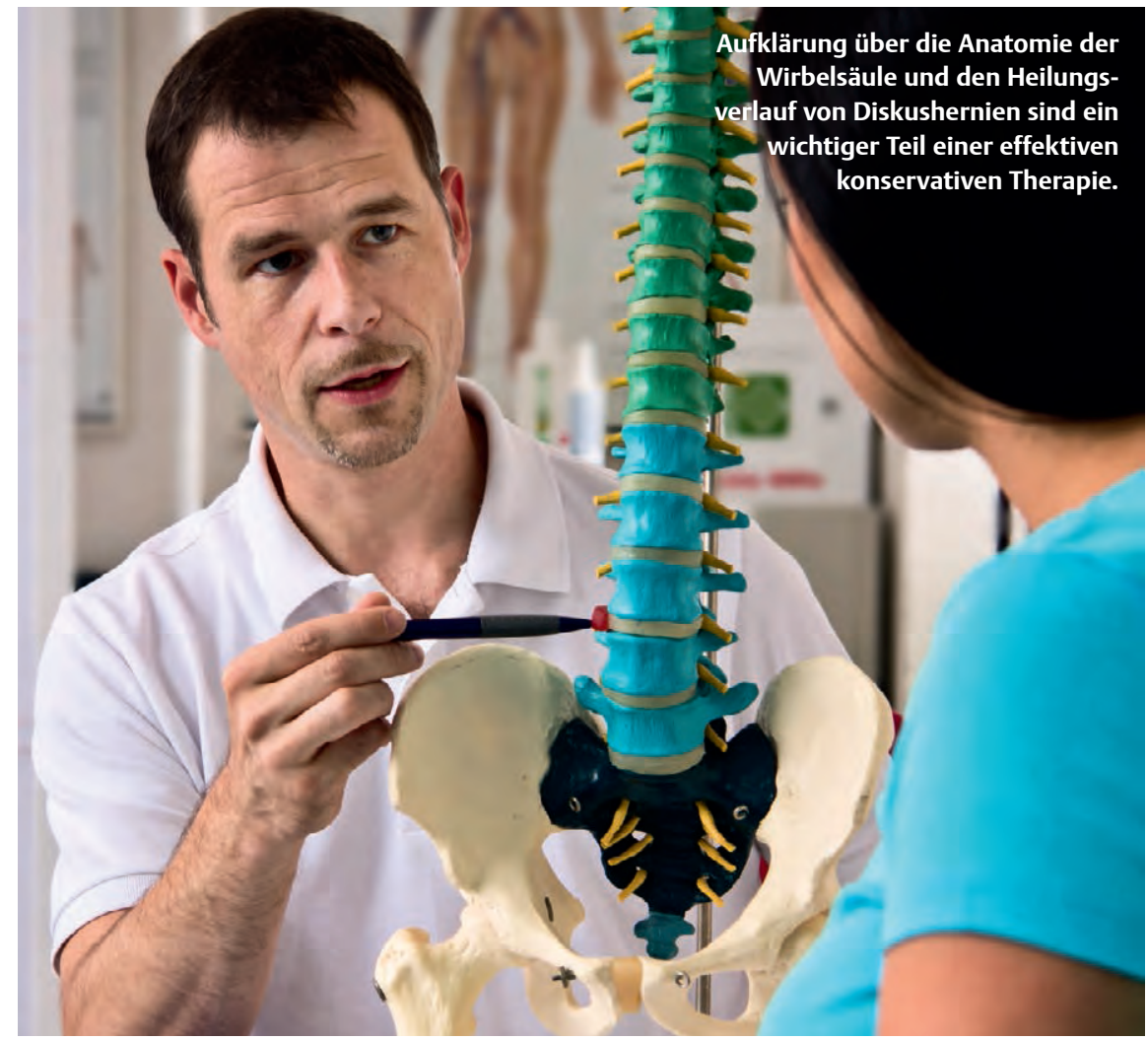

LUMBOISCHIALGIE

\section{Es ist nicht egal, was man macht}

Eine erfolglose konservative Therapie ist für viele Mediziner ein Grund, Patienten mit Lumboischialgie zu operieren. Doch konservative Therapie ist nicht gleich konservative Therapie. Zwei dänische Wissenschaftler belegten nun, wovon die meisten Physiotherapeuten bereits ausgingen: Der Erfolg hängt maßgeblich von den Inhalten ab.

Die Wissenschaftler schlossen 181 Patienten zwischen 18 und 65 Jahre in ihre Studie ein. Alle hatten ein- oder beidseitige, dermatomale Ausstrahlungen mindestens bis zum Knie. Die Schmerzen waren zwischen zwei Wochen und einem Jahr vorhanden und hatten eine Stärke von mindestens 3 auf einer 10-Punkte-VAS. Die Probanden, die alle bereits eine konservative Therapie hinter sich hatten, wurden in zwei Gruppen randomisiert: Alle wurden in einem Gespräch über die Anatomie der Wirbelsäule sowie den Heilungsverlauf einer Diskushernie aufgeklärt. Anschließend führten die Probanden ein Übungsprogramm durch. Für Gruppe 1 bestand dies aus individuellen Übungen basierend auf dem McKenzie-Konzept sowie spe- ziellen Stabilisationsübungen. Gruppe 2 erhielt allgemeine, niedrig intensive Bewegungsübungen, um die allgemeine Blutzirkulation anzuregen. Alle Probanden bekamen im Schnitt knapp fünf Behandlungen, verteilt auf acht Wochen.

Bei der Auswertung zeigte sich, dass sich alle Teilnehmer statistisch signifikant und klinisch relevant verbessert hatten, unter anderem hinsichtlich ihrer Schmerzen und ihres funktionellen Status. Somit war das Ergebnis zu diesem Zeitpunkt bereits besser als das der jeweiligen konservativen Therapie, die die Probanden vor der Studie erhalten hatten. Übungsgruppe 1 hatte sich zudem in den meisten Ergebnisparametern signifikant mehr verbessert als die Gruppe 2, die das allgemeine Trainingsprogramm absolviert hatte. josc Spine 2012; 37: 531-542

$\Rightarrow$ Unter dem Link www.sygehuslillebaelt. dk/wm345075 > „Exercise program“ bzw. „Sham exercises“ können Sie sich die Übungsprogramme der beiden Gruppen auf Englisch herunterladen. 


\section{Umstrittene OP bringt keinen Nutzen}

Einer umstrittenen Theorie nach können Abflussstörungen in der Vena jugularis, die das Gehirn drainiert, und der Vena azygos, die für den Blutabfluss aus dem Rückenmark zuständig ist, bei manchen Patienten zu einer Multiplen Sklerose führen. Bei der als Liberation Treatment bekannten Operation, die sich auf diese Theorie stützt, werden die Vena jugularis interna oder die Vena azygos mit einem Ballonkatheter aufgeweitet, was den Blutfluss normalisieren und die Betroffenen von ihren Symptomen befreien soll. Diese Art von Operation wurde weltweit bisher an rund 30.000 Patienten durchgeführt, in Deutschland oftmals als Selbstzahlerleistung für mehrere Tausend Euro. Der Nutzen dieser OP wurde bisher allerdings nie in einer randomisierten kontrollierten Studie untersucht. Eine aktuelle Untersuchung bestätigt laut der Deutschen Gesellschaft für Neurologie (DGN) nun die schon lange bestehenden Befürchtungen: Die OP nützt nichts, möglicherweise schadet sie sogar.

In der Studie waren zunächst zehn Patienten mittels Liberation Treatment behandelt worden, ohne dass dabei schwerwiegende Nebenwirkungen aufgetreten waren. Die nächsten 19 Teilnehmer bekamen entweder ein Liberation Treatment oder eine Scheinbehandlung, bei der nur der Katheter in die Halsvene eingeführt wurde. Nach sechs Monaten war der Allgemeinzustand der neun behandelten Patienten generell schlechter als der der zehn aus der Plazebogruppe. Zudem hatten die behandelten Patienten im Schnitt vier Schübe gehabt, die nicht behandelten nur einen. Einige Parameter wiesen auf eine vermehrte Krankheitsaktivität nach der Venoplastie hin.

Prof. Dr. Ralf Gold, Vorstandsmitglied der DGN, sagt dazu: „Eine Anwendung des Verfahrens für Selbstzahler ist in Anbetracht der aktuellen Studienlage absolut inakzeptabel.“

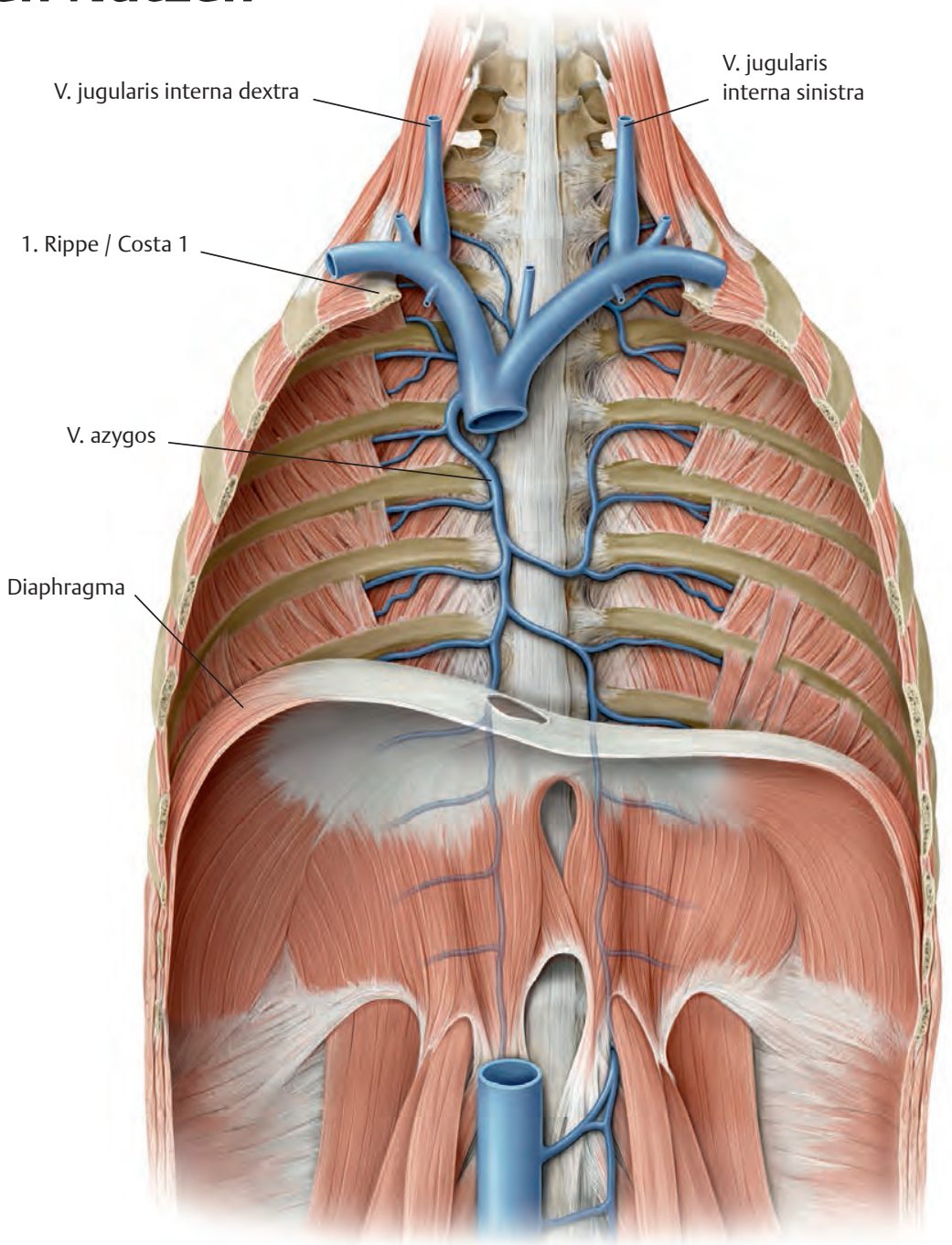

Eine Dilatation der V. azygos oder der V. jugularis interna hat keinen Einfluss auf die Symptome einer Multiplen Sklerose. 


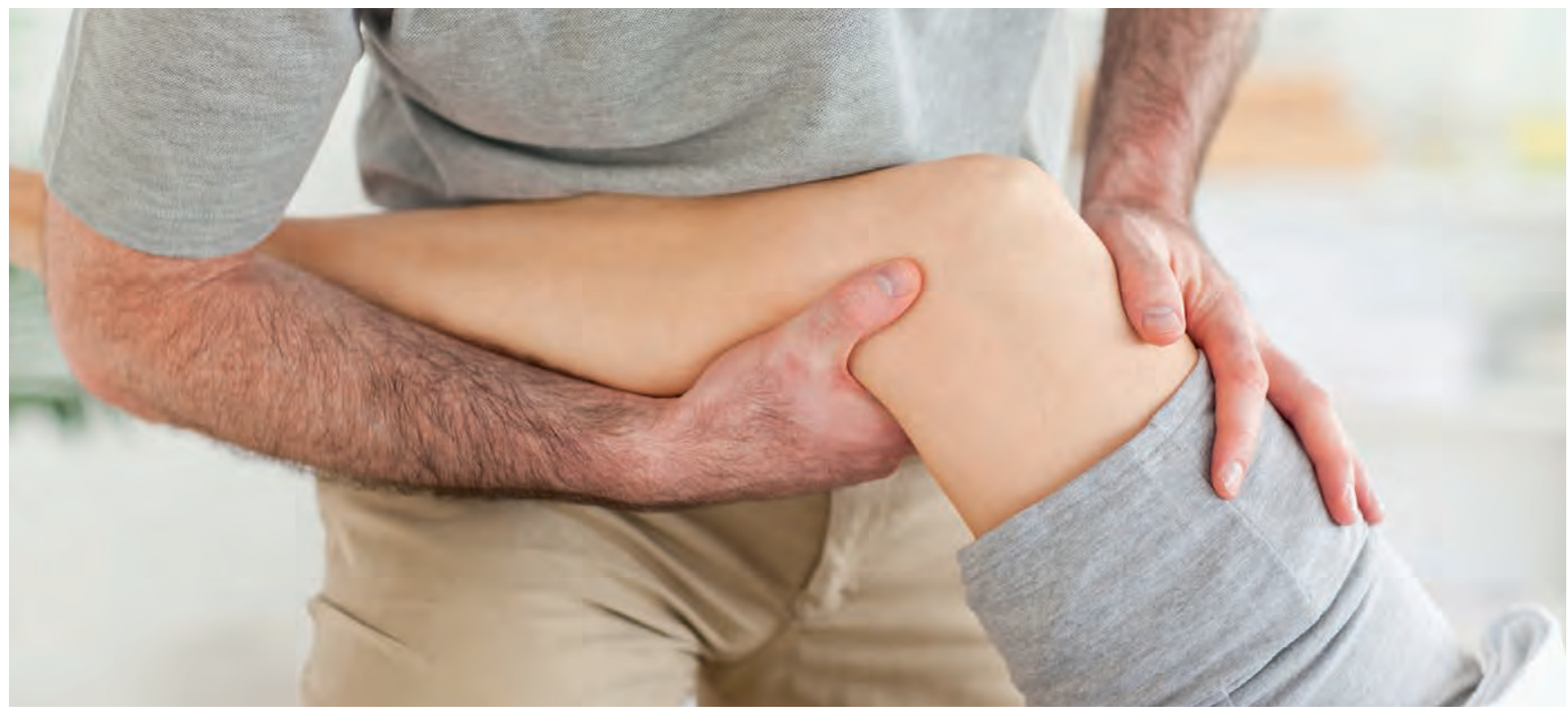

\section{MENISIKUSRISS}

\section{Physiotherapie gleich gut wie Arthroskopie}

Patienten mit Meniskusschaden und Knorpeldefekt im Kniegelenk verbessern sich durch eine Arthroskopie nicht mehr als diejenigen, die ausschließlich physiotherapeutisch behandelt werden. Zu diesem Schluss kommt ein Forscherteam aus den USA.

Es teilte 351 Patienten, die mindestens 45 Jahre alt waren und einen radiologisch nachgewiesenen Knorpeldefekt und Meniskusschaden im Kniegelenk hatten, per Zufall in zwei Gruppen ein: Gruppe 1 wurde operiert und bekam anschließend Physiotherapie.
Gruppe 2 erhielt lediglich Physiotherapie. Denjenigen, die laut Randomisierung ausschließlich Physiotherapie erhielten, stand es während der Therapie frei, sich ebenfalls für eine Operation zu entscheiden. Das Follow-up fand nach sechs und zwölf Monaten statt, primärer Outcome-Parameter war der WOMACScore. Bei diesem Score können Patienten maximal 100 Punkte erreichen - je höher die Punktzahl, desto gravierender die Symptome. Nach sechs Monaten waren die Ergebnisse beider Gruppen ähnlich: Die operierten Stu- dienteilnehmer hatten sich im WOMAC im Schnitt um knapp 21 Punkte verbessert, die Probanden in der Physiotherapie-Gruppe um 18,5. Die Ergebnisse nach zwölf Monaten glichen denen nach sechs Monaten. Allerdings hatten sich rund 30\% derjenigen Patienten, die primär konservativ therapiert wurden, während der ersten sechs Monate für eine OP entschieden.

New Engl J Med 2013; doi: 10.1056/NEJMoa1301408

\section{RÜCKENSCHMERZEN}

\section{„Echte“ Akupunktur effektiver als Plazebo}

Es ist eine ständige Diskussion, ob Akupunktur tatsächlich aufgrund der korrekt gesetzten Nadeln wirkt oder ihr Erfolg „nur“ ein Plazeboeffekt ist. Koreanische Wissenschaftler gingen dieser Frage nun nach.

Sie teilten 130 Erwachsene, die seit über drei Monaten unter Rückenschmerzen mit einer Intensität von mindestens 5 auf einer 10-Punkte-VAS litten, in zwei Gruppen ein. Alle bekamen Übungen und Haltungstipps. Zudem erhielten sie zwölf Akupunktur-Behandlungen. Der Unterschied war, dass die eine Gruppe eine individuelle Akupunktur erhielt, die andere eine Scheinbehandlung. Bei dieser setzten die
Ärzte Nadeln auf Punkte, die keine Akupunkturpunkte waren. Zudem penetrierten die Nadeln die Haut nicht.

116 Patienten standen für die Nachuntersuchung nach drei und zwölf Monaten zur Verfügung. Die Schmerzen in der Interventionsgruppe hatten sich um durchschnittlich 3,36 auf einer 10-Punkte-VAS reduziert, in der Plazebogruppe nur um 2,27 Punkte. Den Probanden der Verumgruppe waren ihre Symptome auch signifikant weniger lästig. Alle anderen Outcome-Parameter verbesserten sich bei allen Probanden gleichermaßen. josc Spine 2013; 38: 549-557 


\section{SKAPULAPOSITION}

\section{Seitenvergleich hinkt}

U Um zu beurteilen, wie die Bewegungsqualität einer von Schmerzen oder sonstigen Pathologie beeinträchtigten Extremität ist, ziehen Physiotherapeuten häufig den Seitenvergleich heran - in der Annahme, die Bewegung sei auf der nicht betroffenen Seite normal. Zwei Forscher aus Portugal überprüften nun, ob die Scapulae gesunder Menschen in verschiedenen Armpositionen tatsächlich beidseits gleich stehen. Denn nur so wäre der Seitenvergleich eine zuverlässige Referenz.

Die Portugiesen rekrutierten dazu 14 gesunde Probanden im Alter zwischen 18 und 26 Jahren. Mithilfe eines dreidimensionalen Bewegungsanalysesystems dokumentierten sie deren Schulterblattposition in drei verschiedenen Armpositionen, und zwar der Ru-

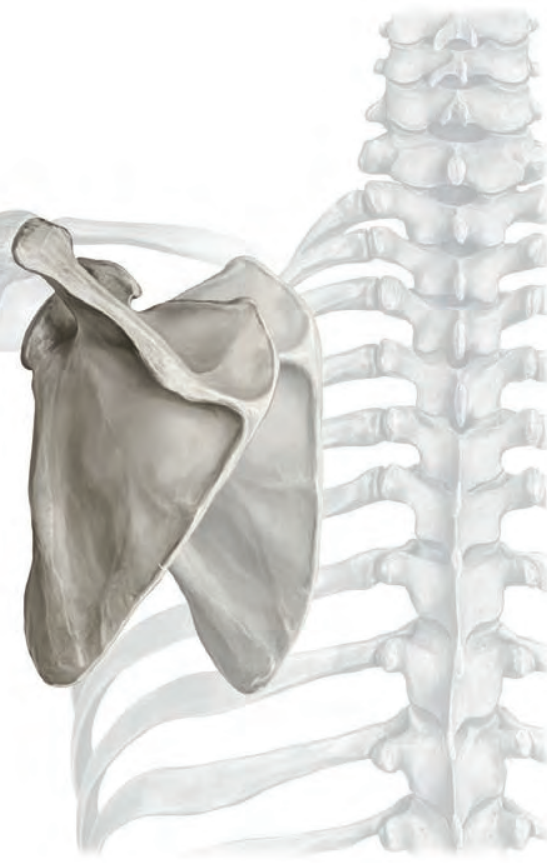

hestellung, mit den Händen an den Hüften sowie mit zur Seite gestreckten Armen. Ergebnis: Die Scapulae standen bei allen Probanden unterschiedlich. Das Schulterblatt der dominanten Hand war in allen drei Positionen mehr retrahiert und aufwärtsrotiert als das der anderen Seite. Somit kann bei einer Schulterpathologie die Skapulaposition der nicht betroffenen Seite nur bedingt als Referenz dienen.

josc

Man Ther 2013; 18: 46-53

\section{HWS-RADIKULOPATHIE}

\section{Physiotherapie macht OP überflüssig}

a Patienten, die aufgrund einer HWS-Radikulopathie Physiotherapie erhalten, geht es anschließend deutlich besser - egal, ob sie zuvor operiert wurden oder nicht. Zu diesem Fazit kamen schwedische Wissenschaftler in einer randomisierten Studie mit zwei Jahren Follow-up.

An der Untersuchung nahmen 63 Patienten teil, die seit mindestens acht Wochen und höchstens fünf Jahren an einer bandscheibenbedingten Nervenwurzelkompression in einem oder zwei HWS-Segmenten litten. Die Diagnose wurde mittels MRT gestellt und musste mit der klinischen Untersuchung korrelieren. Die Teilnehmer wurden in zwei Gruppen randomisiert. Gruppe 1 bekam über vierzehn Wochen hinweg zweimal wöchentlich ein Physiotherapieprogramm. Es beinhaltete unter anderem Nackenstabilisationsübungen, Haltungskorrektur, Thoraxmobilisationen und Aufklärung über Schmerzmechanismen. Gruppe 2 wurde zunächst an der HWS operiert: Die Operateure dekomprimierten die betroffene Nervenwurzel und fusionierten anschließend das Segment. Drei Monate später begannen diese Probanden mit dem gleichen Programm wie Gruppe 1.

Die Wissenschaftler stellten fest, dass sich beide Gruppen im Follow-up nicht signifikant unterschieden. Daher schlagen sie vor, dass Patienten mit HWS-Radikulopathie zunächst ein strukturiertes physiotherapeutisches Behandlungsprogramm absolvieren, bevor sie operiert werden. 


\section{physiowissenschaft}

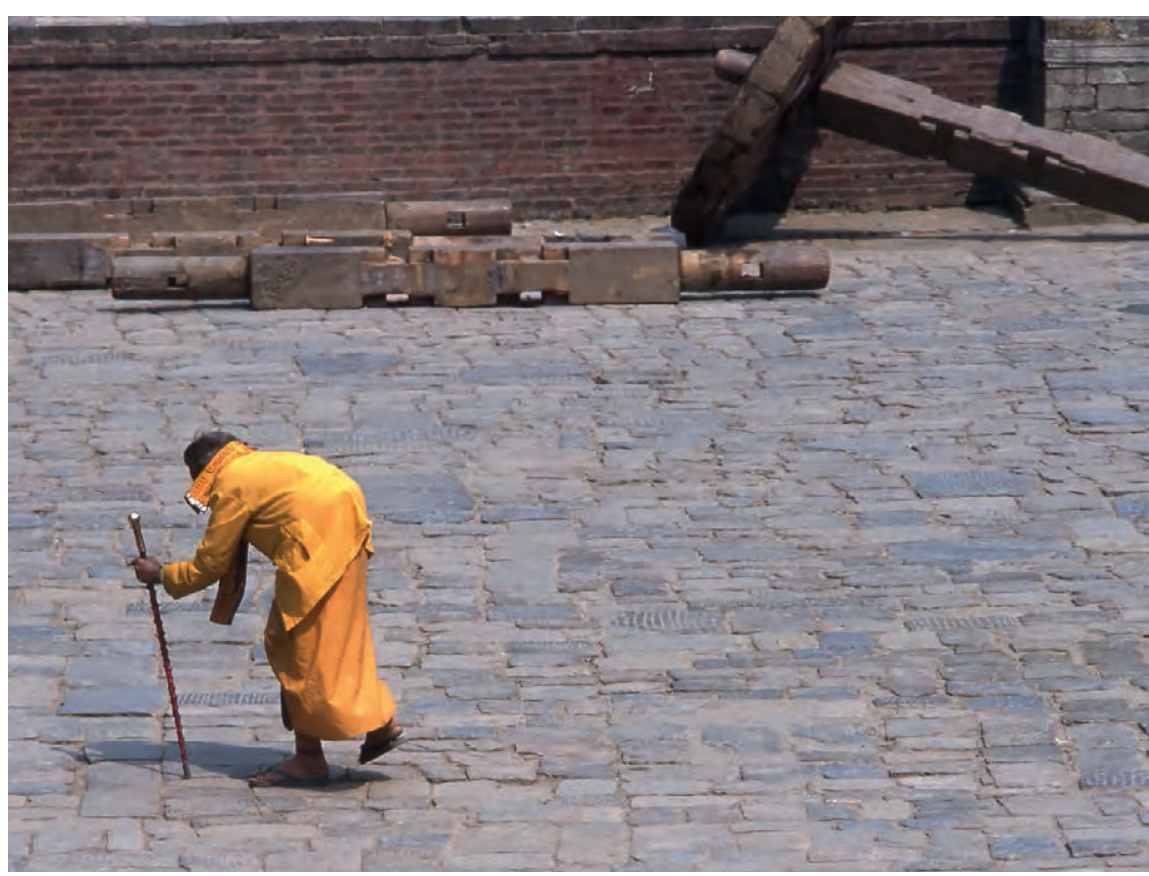

\section{MORBUS BECHTEREW}

\section{Fitnesstraining notwendig}

Kommen Patienten mit Morbus Bechterew zur Physiotherapie, steht meist allein die Beweglichkeit im Mittelpunkt der Therapie - zu Unrecht. Denn auch die kardiorespiratorische Fitness dieser Patienten scheint oft merklich reduziert zu sein.
> allgemeine Beweglichkeit mittels des „Bath Ankylosing Spondylitis Metrology Index“ (BASMI)

$>$ muskuläre Kapazität anhand der maximalen Zahl an Liegestützen

$>$ motorische Kontrolle mittels Achterfigurgehen und Einbeinstand

Diese Werte verglichen die Forscher mit der Krankheitsaktivität, die durch den Ankylosing Spondylitis Disease Activity Score (ASDAS) bestimmt wurde. In diesen fließen unter anderem Morgensteifigkeit, Rückenschmerzen und CRP-Wert (ein unspezifischer Entzündungswert) ein. Zusätzlich dokumentierten die Norweger, wie häufig und wie stark die Patienten körperlich aktiv waren und welche Funktionseinschränkungen sie hatten.

Sie stellten fest, dass die Teilnehmer mit Morbus Bechterew eine klinisch relevante, signifikant schlechtere kardiorespiratorische Fitness hatten als die Gesunden. Erwartungsgemäß war auch ihre Beweglichkeit signifikant schlechter, die muskuläre Kapazität und die motorische Kontrolle unterschieden sich dagegen nicht. Die Krankheitsaktivität korrelierte invers mit der kardiorespiratorischen Fitness: Je unfitter die Patienten waren, desto höher war ihre Krankheitsaktivität.

josc

Phys Ther 2012; 92: 298-309 